\title{
Determining responders and non-responders to fish consumption
}

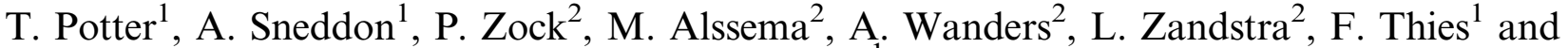 \\ B. de Roos ${ }^{1}$ \\ ${ }^{1}$ Rowett Institute, Foresterhill, University of Aberdeen, Ashgrove Road West, Aberdeen, AB25 2ZD and \\ ${ }^{2}$ Unilever Research and Development Vlaardingen B.V., Olivier van Noortlaan 120, 3133 AT Vlaardingen, The \\ Netherlands ("Unilever")
}

The FISH DISH study examined the effect of eating two $140 \mathrm{~g}$ portions per week of salmon with either a high (HPUFA) or moderate (SPUFA) content of omega-3 fatty acids, compared with eating no salmon $(\mathrm{CONTROL})$, for 18 weeks in 51 healthy volunteers $(\mathrm{n}=$ 17/group). We measured 168 outcomes assessing heart health, micronutrient status, inflammation, oxidative stress and gut health after 9 and 18 weeks of intervention. We found that consumption of both salmon types significantly increased participants' Omega-3 Index (O3I: the amount of eicosapentaenoic acid (EPA) and docosahexaenoic acid (DHA) as a ratio of total fatty acids incorporated in erythrocyte membranes) from $5.9 \%$ to $8.0 \%(p<0.05)$ (Figure 1). Consumption of SPUFA salmon also significantly decreased plasma triglycerides $(p<0.05)$ (Figure 1$)$, which is in line with the observed triglyceride-lowering effects of fish oil ${ }^{(1)}$. However, we observed considerable variation in responsiveness between individuals in both salmon groups (Figure 1).
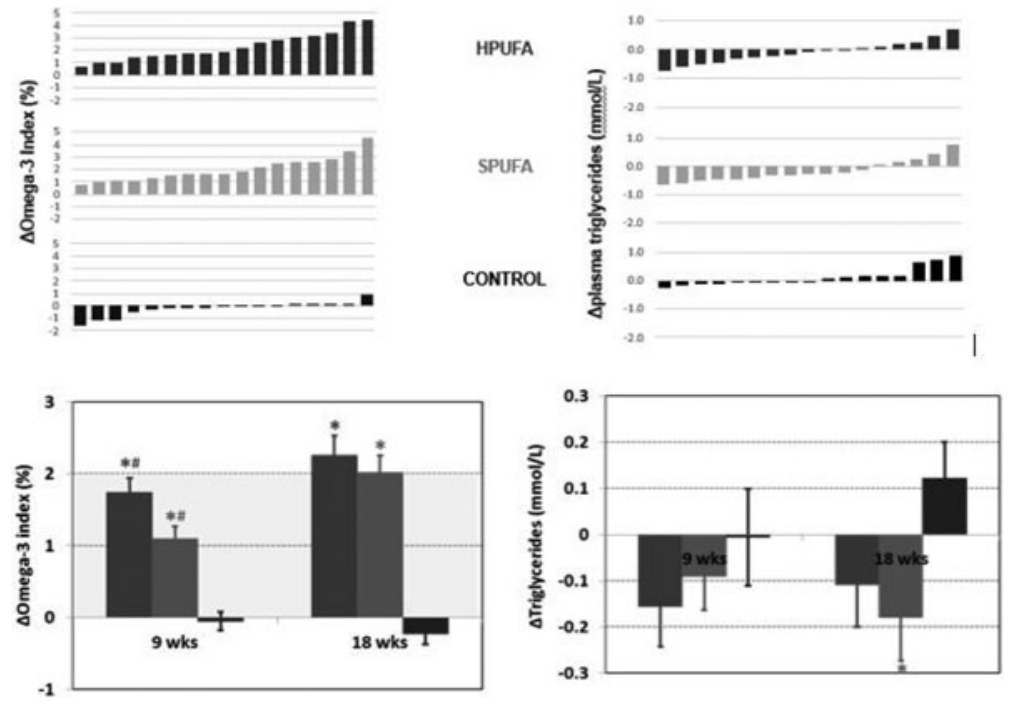

Fig. 1.

We designed and executed an analysis pipeline to consider individual responsiveness to dietary intervention using $\mathrm{R}$ software. This analysis investigated whether (1) baseline markers and measures of dietary intake could predict O3I and triglyceride response to intervention using K-mean clustering; and (2) we could define "high" and "low" responders to assess whether both groups could be differentiated based on baseline data. Clusters were compared using one-way ANOVA. Responders and non-responders were compared using t tests. Relationships between variables were analysed using simple and multiple linear regression.

K-means clustering on baseline data did not predict the magnitude of response in the O3I and plasma triglycerides, despite one cluster having a much higher O3I at baseline. However, we found that lower levels of plasma p-Selectin at baseline were linked to a higher decrease in plasma triglycerides over the course of the study in both salmon groups $(\mathrm{p}<0.05)$. This suggests that baseline inflammation may affect the individual triglyceride response upon consumption of oily fish.

When comparing individual responses in O3I that were either above ("responders") or below the median ("non-responders"), we found that only in the group consuming salmon containing moderate levels of omega-3 fatty acids, those whose O3I increased above the median had a significantly lower energy intake at both the start and end of the study. This effect was seen across both sexes $(p=0.01)$. When comparing the top 6 responders with the bottom 6 non-responders in terms of O3I, the 'high responders' were found to have a higher protein intake as $\%$ of daily energy intake $(p=0.02)$. This suggests there may be a link between energy/protein intake and response in O3I.

This precision nutrition-style analysis framework has been shown to reveal valuable insights into the main drivers of responsiveness, and may help to evaluate which diet works for whom in future studies ${ }^{(2)}$.

Funded by BBSRC, Unilever and the Scottish Government Rural and Environmental Science \& Analytical Services Division.

1. Mozaffarian D, Rimm E (2006) JAMA 296, 1885.

2. de Roos B, Brennan L (2017) Nutrients 9, 847. 